\title{
Detached Eddy Simulations of an Airfoil in Turbulent Inflow
}

\author{
Lasse Gilling* \\ Aalborg University, 9000 Aalborg, Denmark \\ Niels N. Sørensen ${ }^{\dagger}$ \\ National Laboratory for Sustainable Energy, Risø-DTU, Technical University of Denmark, \\ 4000 Roskilde, Denmark \\ Lars Davidson $\ddagger$ \\ Chalmers University of Technology, 412 96 Gothenburg, Sweden
}

\begin{abstract}
The effect of resolving inflow turbulence in detached eddy simulations of airfoil flows is studied. Synthetic turbulence is used for inflow boundary condition. The generated turbulence fields are shown to decay according to experimental data as they are convected through the domain with the free stream velocity. The subsonic flow around a NACA 0015 airfoil is studied at Reynolds number $1.6 \times 10^{6}$ and at various angles of attack before and after stall. Simulations with turbulent inflow are compared to experiments and to simulations without turbulent inflow. The results show that the flow is sensitive to the intensity of the resolved turbulence. Especially, when the flow is close to stall, separation can be triggered if the turbulence is resolved. By resolving the inflow turbulence better agreement with experimental data can be achieved.
\end{abstract}

\section{Introduction}

Wind turbines operate in the atmospheric boundary layer and are consequently subjected to the effects of inflow turbulence. In this paper the effect of resolving the free stream turbulence in simulations on airfoils is investigated. Only an airfoil section will be analyzed to keep the computational costs at realistic levels.

To resolve the upstream turbulence the simulation technique must be large-eddy simulation (LES) or direct numerical simulation (DNS). LES is chosen to limit computational costs, as DNS is infeasible at large Reynolds numbers. Resolving the largest eddies in the boundary layer at Reynolds numbers in the order of millions is prohibitively computationally expensive. Consequently, a form of wall modeling is applied to reduce the cost. A hybrid of LES and the Reynolds averaged Navier-Stokes (RANS) equations is chosen to accurately predict the flow under massive separation.

Detached-eddy simulation (DES) is chosen, as it is an elegant form of hybrid LES/RANS. It was developed for simulating flows around airplane wings. ${ }^{1}$ Since then, the technique has successfully been applied to flows around airfoil sections. ${ }^{2-4}$ DES has also been applied to an entire wind turbine blade. ${ }^{5}$

Neither of the above references attempt to resolve the inflow turbulence. This allows for a coarse grid upstream of the airfoil, as the flow is laminar in this region. In contrast, the upstream turbulence is resolved in this work, which leads to the need for a fine mesh upstream of the airfoil.

The effects of resolving the upstream turbulence on a NACA 0015 airfoil flow at Reynolds number $1.6 \times 10^{6}$ will be analyzed. Data from experiments conducted in the LM wind tunnel ${ }^{6}$ is used for comparison. A brief description of the experimental setup and the obtained data is given by Døssing ${ }^{7}$ and Bove. ${ }^{8}$

In this paper results from simulations will be validated against the experimental data. The lift, drag and surface pressure coefficients are compared to experiments. Further, the simulation time required for

\footnotetext{
*Ph.D.-student, Department of Civil Engineering, Sohngaardsholsvej 57, AIAA Member

†Senior Scientist, Wind Energy Division, Frederiksborgvej 399, P.O. Box 49, AIAA Senior Member

‡Professor, Division of Fluid Dynamics, Department of Applied Mechanics, Hörsalsvägen 7A
} 
flow development and averaging will be discussed. Finally, the resolved stresses and the eddy viscosity from a simulation are shown. Prior to presenting the numerical setup and the results obtained, the numerical methods are described and a discussion on the inflow boundary condition is given.

\section{Numerical Methods}

The in-house computational fluid dynamics code EllipSys3 $3 \mathrm{D}^{9-11}$ is used for the flow simulations. The code is a multiblock finite volume discretization of the incompressible RANS equations in general curvilinear coordinates for structured meshes. The code uses a collocated variable arrangement, and Rhie/Chow interpolation is used to avoid odd/even pressure decoupling. The PISO algorithm is used to enforce pressure/velocity coupling. Solution for momentum is obtained by the fourth order central difference scheme and a second order accurate dual time stepping algorithm is used. The code is parallelized with MPI.

The present version of DES ${ }^{2}$ uses the two-equation $k-\omega$ SST subgrid model. ${ }^{12}$ Application of DDES ${ }^{13}$ is not required for the present Reynolds number and resolution of the boundary layer around the airfoil.

\section{Generation of Synthetic Turbulent Inflow}

The upstream boundary-layer at wind tunnel walls, screens, etc. will not be resolved to minimize computational costs. This section describes the chosen method to generate the inflow turbulence synthetically. The turbulence imposed on the inflow boundary condition should have the correct moments and spectra and appropriate phase angle between modes. Since there are no walls upstream of the airfoil a wind field with the correct moments and spectra can be generated synthetically from a theoretical spectrum. The applied method ${ }^{14}$ only gives correct moments up to second order, and further, the phase information is chosen randomly. Therefore, a precursor simulation is run to let the flow solver correct these deficiencies.

\section{A. The Mann Method}

An entire family of methods originates from the paper by Shinozuka and Jan, ${ }^{15}$ including the method of $\mathrm{Mann}^{14}$ which is used here. The method was designed for generating a turbulent wind field for engineering load calculations. It is based on three-dimensional FFT of a set of wave number vectors determined to give the correct covariance. This results in a divergence free velocity field with the correct spatial correlation. The generated velocity field is three-dimensional in space, and it is converted to a time series of inflow boundary conditions by application of Taylor's hypothesis. All three components of the velocity vectors can be generated. This is advantageous, compared to the option of only generating the streamwise components. This will be shown in the following section.

\section{B. Demand for Three-Dimensional Velocity Fields}

The airfoil should experience homogenous, isotropic turbulence. Some techniques for generating turbulent inflow only generate the streamwise velocity component and let the two remaining components be generated by the flow solver. This approach requires a long upstream domain or a long precursor simulation to allow the velocity field to become isotropic. If all three components are generated synthetically and imposed on the inflow boundary the problem diminishes as shown below. In Figure 1 the isotropy of the velocity field is compared for the two methods.

The results presented in Figure 1 are generated by two simulations with identical computational domains, but different inflow conditions. The domain has $1024 \times 32 \times 32$ cubic cells and periodic boundary conditions in the transverse directions. The two sets of inflow conditions contain the same kinetic energy. One contains three components with the energy evenly distributed, whereas the other contains all the energy in the streamwise component.

As seen from the figure, when the inflow boundary condition only includes one component, the turbulence does not become isotropic in this domain, even though it has a considerable extent in the streamwise direction. In conclusion the inflow boundary condition should contain all three components of the turbulence to minimize the required length of the precursor domain. 


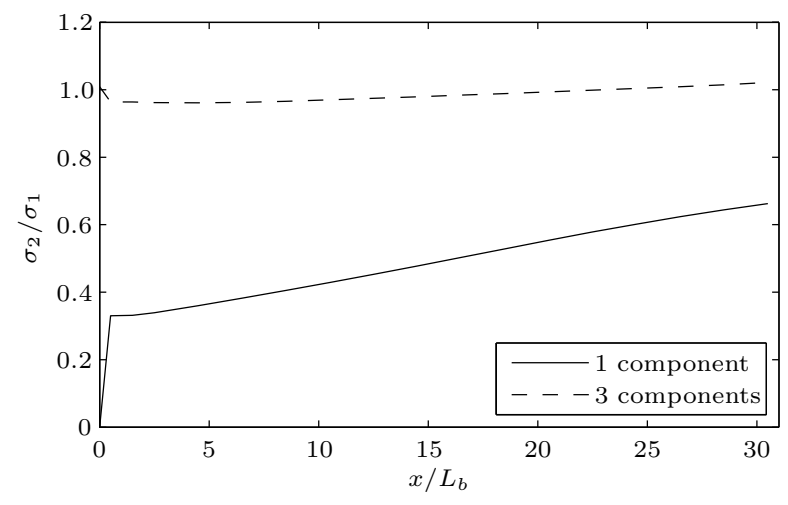

Figure 1. Development of the ratio between the standard deviation of a transverse velocity component and the streamwise velocity component. One simulation has all three velocity components in the inflow, whereas the other only has the streamwise component. $\mathrm{x}$ is the streamwise coordinate measured from the inflow boundary, and $L_{b}$ is the width and height of the domain.

\section{Application: Spatially Decaying Homogenous Turbulence}

Spatial decay of homogenous turbulence is studied to test the synthetic turbulence and the numerical method. The computational domain is illustrated in Figure 2, where the first fifth in the streamwise direction is shown. The domain consists of $1280 \times 64 \times 64$ cubic cells. The mean velocity is constant and oriented along the $x_{1}$ axis. Synthetic turbulence is superimposed on to the constant mean velocity and set as inflow boundary condition. Below the development of resolved turbulent kinetic energy and the energy spectrum is studied.

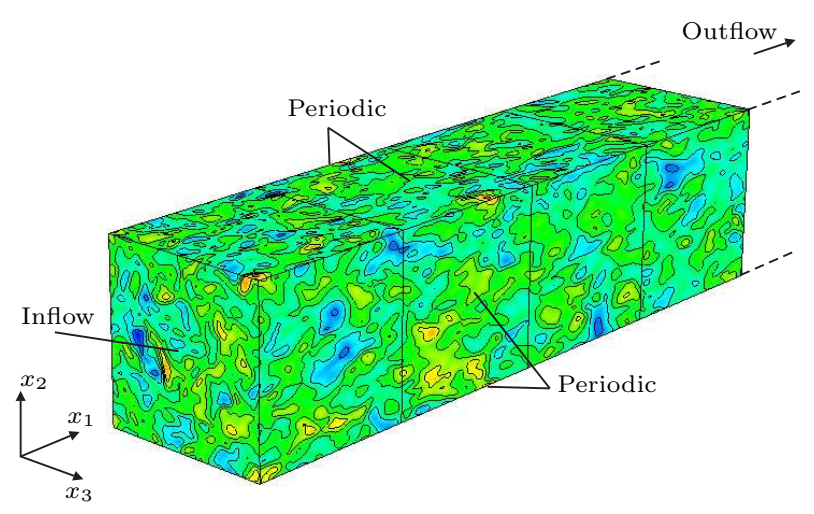

Figure 2. The first fifth of the precursor domain. The boundaries are colored by the streamwise velocity component.

When the synthetic inflow boundary condition is imposed on a computational domain, the turbulence does not decay physically in the first part of the domain. There are two reasons for this. One is that the appropriate values of the subgrid model parameters (here $k$ and $\omega$ ) are not known, and therefore, uniform constant values are imposed on the boundary. The other reason is that the synthetic turbulence does not possess the correct phase information, as the phase angles are chosen randomly. During the precursor simulation these flaws are corrected by the flow solver. ${ }^{16}$

In Figure 3 the decay of resolved turbulent kinetic energy is shown and compared to the empirical expression $^{17}$

$$
k(t)=\frac{1}{2} U_{0}^{2}\left(\frac{1}{21}+\frac{2}{20}\right)\left(\frac{U_{0} t}{M}-3.5\right)^{-1.25}
$$

where $U_{0}$ is a reference velocity and $M$ is the mesh spacing in the grid used to generate the turbulence. The numerical constants have been fitted to match the experimental data.

The decay is shown for three cases, (a), (b) and (c). The inflow in case (a) is synthetic turbulence with the same resolved kinetic energy and integral length scale as the experiment. At some $x_{1}$ station the velocities 


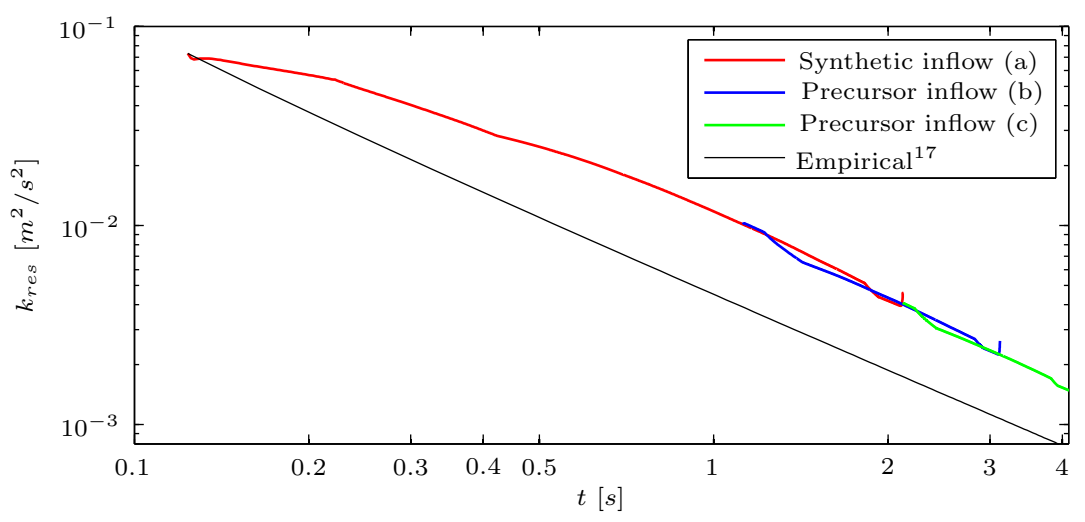

Figure 3. Decay of resolved turbulent kinetic energy.

and subgrid parameters are sampled in the $x_{2}-x_{3}$-plane and the time series of samples are stored to a file. The values sampled from case (a) are used as time dependent inflow boundary condition for case (b). Again, the flow is sampled at some $x_{1}$ and the samples from case (b) are used as inflow boundary condition for case (c).

When the synthetic turbulence is used for the inflow boundary condition the decay is initially too slow, which causes the curve for case (a) to lie above the line from the empirical expression. This is due to a too low eddy viscosity on the inflow boundary. It is observed that the two last cases behave differently from the first but similarly to each other. The curve for case (b) starts at the point on curve (a) from where its inflow turbulence is sampled. From there it makes a kink before it starts to decay with the correct rate; the curve is parallel to the empirical line. The curve for case (c) starts from a point on curve (b) and it also makes the kink, before it starts to decay with the correct rate.

The velocities and subgrid parameters are set on the inflow boundary, so the only variable which is not identical to the values in the precursor simulation is the pressure. The incorrect pressure may cause the kink in the kinetic energy decay seen in the two last cases. Close to the outlet the outflow boundary condition cause the decay to be incorrect and another kink can be seen on all curves.

The energy spectra from time series sampled at different locations in the three simulations are shown in Figure 4. By Taylor's hypothesis the sample positions are converted to instances as in Figure 3. Only a short range of frequencies can be resolved due to the dimensions of the computational domain. The width of the domain sets the lower limit and the size of the cells set the upper one. The domain consists of only 64 cells in the transverse directions, and as seen in the figure it can only resolve a decade of frequencies. It can be seen that the largest eddies in the inertial subrange are resolved as the slopes of the spectra computed from the simulations are $-5 / 3$ for high frequencies.

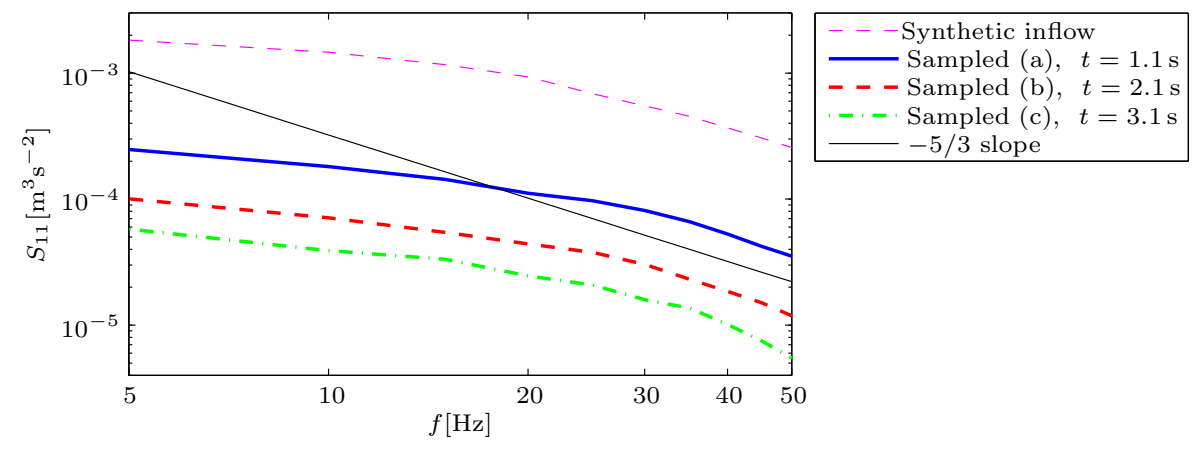

Figure 4. Energy spectra of turbulence sampled at three positions in the streamwise direction. 


\section{Computational Setup}

The mesh, boundary conditions and scaling of the inflow turbulence will be described below.

\section{A. Computational Domain}

The geometry of the domain has been made corresponding to the geometry of the wind tunnel where the experiments are conducted. The mesh is shown in Figures 5 and 6. It is a two dimensional mesh which has been extruded in the spanwise direction to make a three dimensional mesh. It consists of an O-mesh near the airfoil and Cartesian blocks to fill the rest of the domain to match the geometry of the wind tunnel. The Cartesian grid in large parts of the domain is favorable for the energy conservation. The O-mesh consists of $384 \times 64$ cells in the wall-parallel and wall-normal directions, respectively. The spanwise direction is resolved by 128 cells of constant width. The mesh consists of a total of 21 million cells.

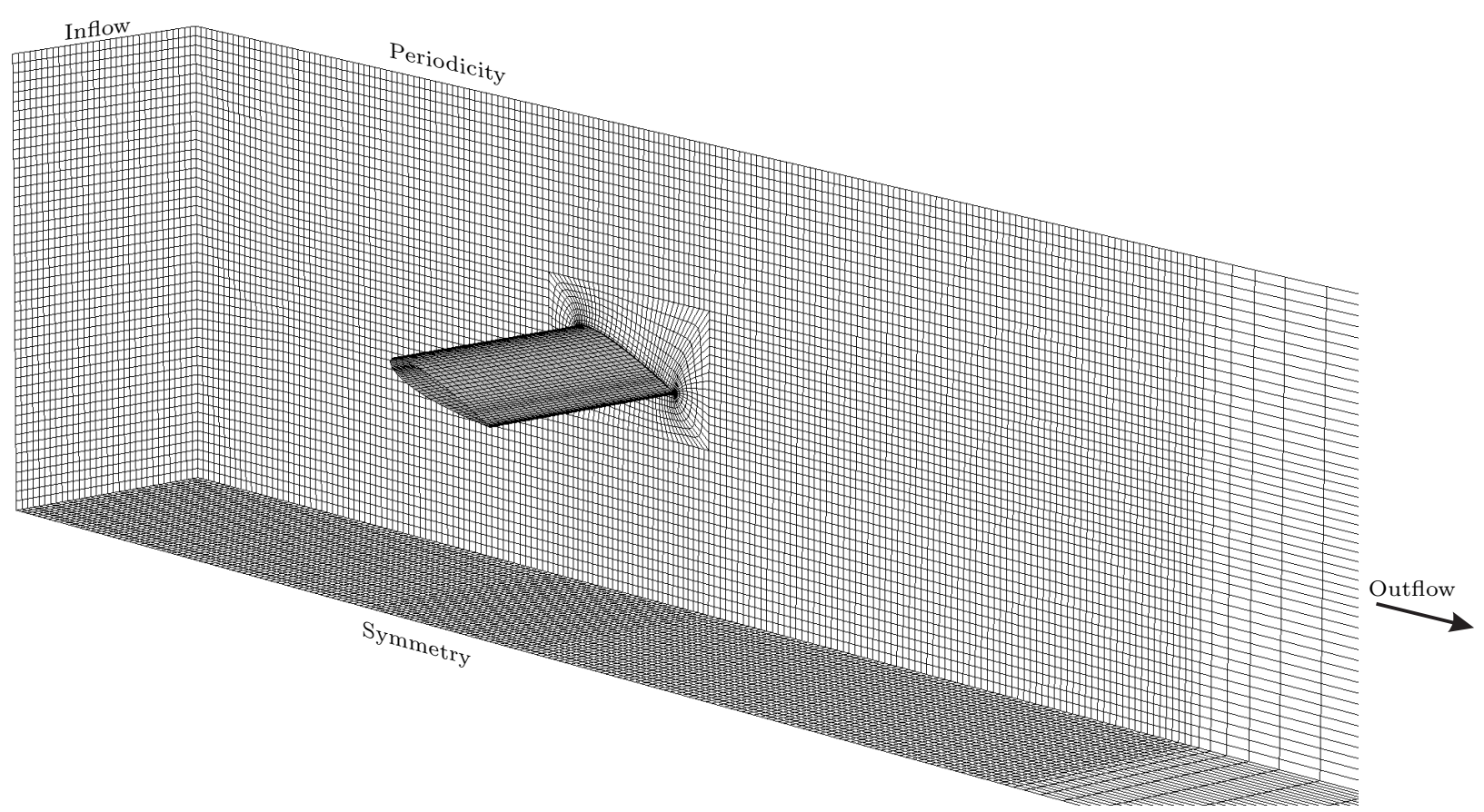

Figure 5. Three-dimensional view of grid. Only every fourth mesh point is shown. The angle of attack in the shown mesh is $15^{\circ}$.

Symmetry boundary conditions are used on the top and bottom of the domain to limit the flow without having to resolve a wall boundary layer. For the same reason periodic boundary conditions are imposed on the spanwise boundaries. Velocities, $k$ and $\omega$ taken from a precursor simulation are used as time dependant inflow boundary condition. On the outflow boundary the gradient is assumed to be zero normal to the outlet, except for pressure which uses a second order extrapolation normal to the boundary.

As in the experiment the spanwise extent of the domain is 1.5 chords and the height is 3 chords. The inflow boundary is located 4 chords upstream and the outflow boundary is located approximately 25 chords downstream of the airfoil. The last 64 cells in the streamwise direction are stretched to suppress resolved turbulence prior to the outflow boundary. The stretching ratio is $5 \%$.

The size of the cells in the Cartesian mesh (excluding the stretching region) is $\Delta x \times \Delta y \times \Delta z \approx 1.4 \times 1.6 \times 1.2$ when measured in percent of the chord. $x, y$ and $z$ denote the streamwise, vertical and spanwise directions, respectively. In Figure 7 the size of the cells closest to the airfoil wall is shown. The mesh spacing has been normalized to wall units for the $15^{\circ}$ angle of attack (AOA) flow. $\Delta n^{+}$and $\Delta s^{+}$are mesh spacings in the wall-normal and wall-parallel directions, respectively. $\Delta z$ is constant but $\Delta z^{+}$varies due to changes in the friction velocity. The ratio of $\Delta n^{+}$and $\Delta z^{+}$is nearly constant, as $\Delta n$ is nearly constant.

In Sørensen ${ }^{18}$ the mesh dependance was investigated for a rotor simulation using the QUICK scheme in the EllipSys3D code. Decent mesh independence was shown for a mesh of similar resolution in the $n$ and $s$ directions and a much coarser resolution along the spanwise direction. 




Figure 6. Close-up of the two dimensional O-mesh and some neighboring Cartesian cells. The angle of attack in the shown mesh is $15^{\circ}$.

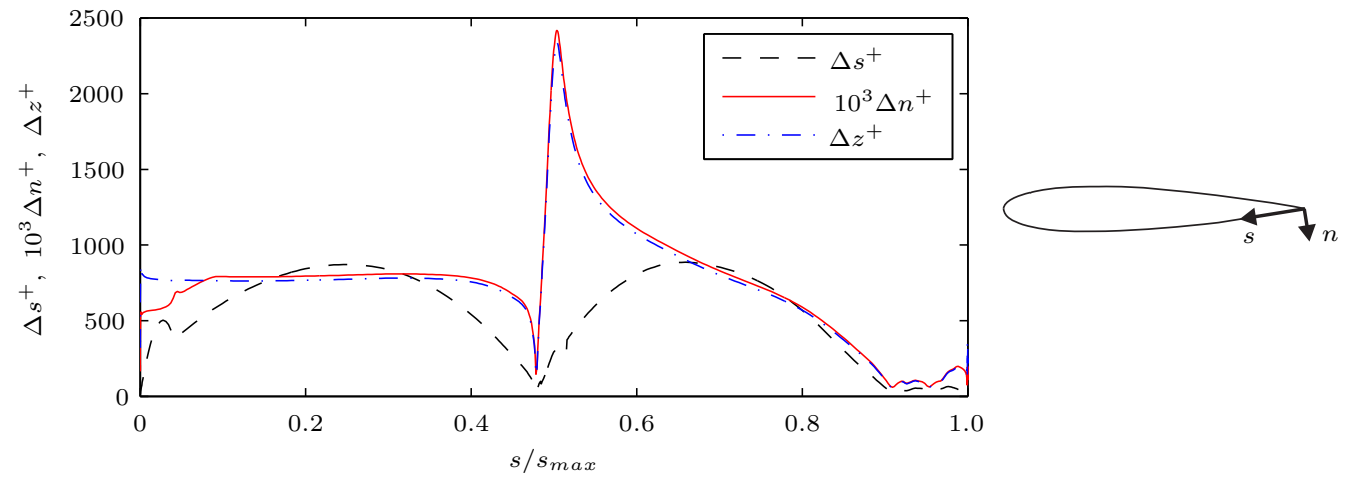

Figure 7. Left: Length, height and width the of first layer of cells measured in wall units. Right: Definition of coordinates $s$ and $n$.

\section{B. Scaling of the Inflow Boundary Condition}

The intensity of the inflow turbulence is varied by scaling the velocities and subgrid scale model parameters, which have been sampled from a precursor simulation. Let $u_{0}^{\prime}, v_{0}^{\prime}, w_{0}^{\prime}, k_{0}^{\prime}$ and $\omega_{0}^{\prime}$ denote the values sampled from a precursor simulation. The velocities are scaled by a common factor, $a$, to set a given intensity of the resolved inflow turbulence. $k_{0}^{\prime} \propto\left(u_{0}^{\prime}\right)^{2}$ is scaled by $a^{2}$ and $\omega_{0}^{\prime} \propto \sqrt{k} / L$ is scaled by $a$. Hence, the length scale of both the resolved and subgrid turbulence is constant for all the applied inflow turbulence fields. The resolved turbulence has an integral length scale of approximately 0.25 chords. The above relations for scaling the subgrid parameters have been validated by studying the decay of the resolved turbulence and subgrid parameters in a precursor simulation.

The imposed eddy viscosity scales linearly with the factor $a$. It ranges from about $0.7 \nu$ for a resolved turbulence intensity (TI) of $0.1 \%$ to $14 \nu$ for $2.0 \%$ TI. $\nu$ is the molecular viscosity. In the simulations without resolved inflow turbulence constant values of $k$ and $\omega$ are set on the inflow boundary to give an eddy viscosity of about $10^{-3} \nu$ just upstream of the airfoil.

The length of the time series sampled from the precursor simulation corresponds to 9 chord passages. To run simulations longer than this the same inflow field is simply imposed a number of times. 


\section{Results and Discussion}

In this section the simulations are compared to the experiment. Initially, the time averaged values of lift, drag and surface pressure are presented. Then, some observations on the transient nature of the flows are given. Finally, the details of resolved stresses and eddy viscosity are investigated in greater detail for the flow at $12^{\circ} \mathrm{AOA}$.

\section{A. Time Averaged Lift, Drag and Pressure Distribution}

In Figure 8 the computed lift and drag coefficients are compared to data from the experiment by LM Glasfiber. ${ }^{8}$ The $\mathrm{TI}$ in the wind tunnel is in the order of $0.1 \%$ and in the experiment the flow stalls close to $15^{\circ}$ AOA. During the experiment both positive and negative AOA were tested. As the airfoil is symmetric, the data from negative AOA have been mirrored to positive AOA to give an impression of the accuracy of the experimental data. A small lack of symmetry in the measurements can be seen from the differences in lift coefficient measured at positive and negative AOA. The wind tunnel of LM Glasfiber has been optimized for Reynolds numbers in the range 3-6 million and in this range the results are symmetric.
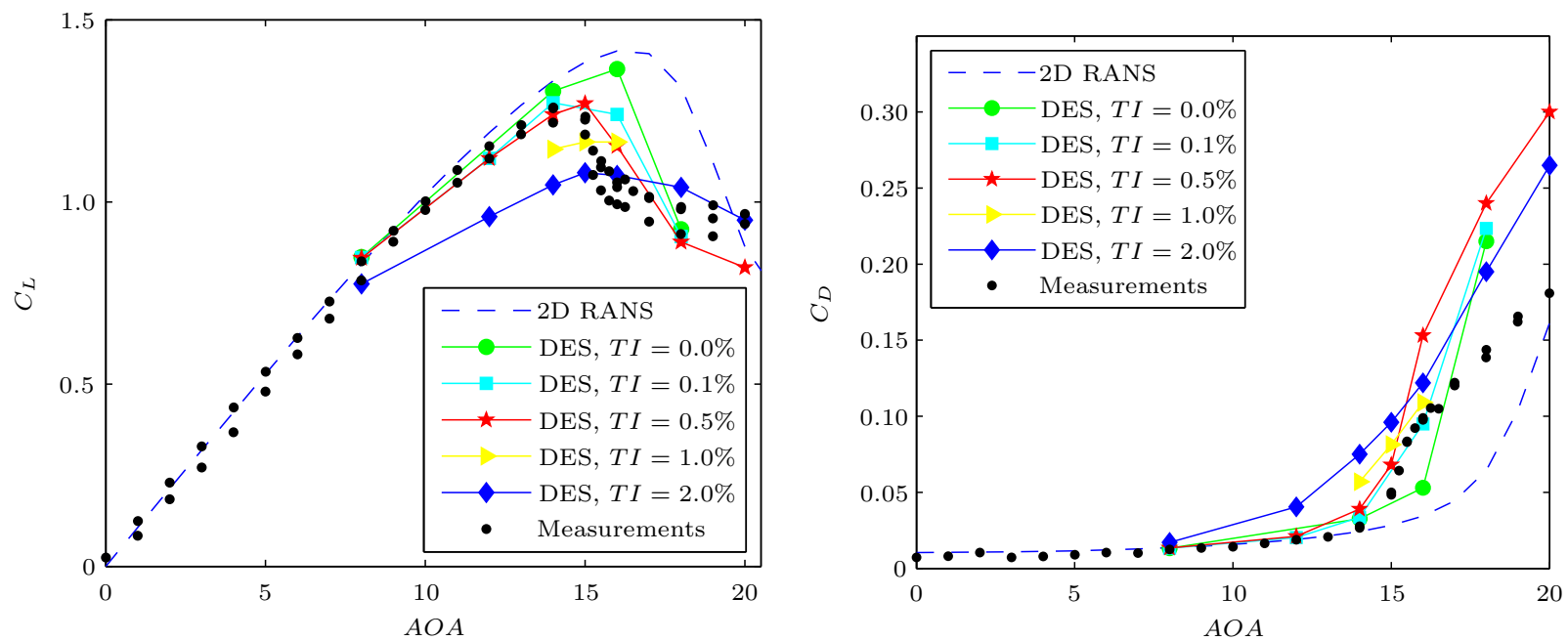

Figure 8. Lift and drag coefficients from simulations and measurements.

In Figure 9 the computed surface pressure coefficient is compared to experiments. From the figures it can be concluded that the flow is sensitive to the intensity of the resolved inflow turbulence. The effect is largest close to stall, where a small increase in the intensity of the resolved turbulence can trigger separation.

The simulated surface pressure distribution agrees well with the experiments for angles of attack lower than $18^{\circ}$. For $20^{\circ}$ the agreement is poor, however. The turbulence intensity that gives best agreement with measurements is not constant for all angles of attack. For AOA lower than stall the optimal agreement is achieved with an intensity around $0.1-0.5 \%$. For higher AOA the agreement is better with $T I=2 \%$. For AOA lower than $15^{\circ}$ an increase in the intensity of the resolved inflow turbulence moves the separation point upstream. For higher AOA the turbulence has the opposite effect.

The skin friction coefficient is shown for $14^{\circ}$ and $20^{\circ}$ AOA in Figure 10. The absolute value is plotted due to the difficulty of defining the orientation of the flow on a two dimensional surface. The above mentioned effect of the resolved inflow turbulence on the separation point is clearly seen from the figure. The stagnation point is largely unaffected by the resolved turbulence. 


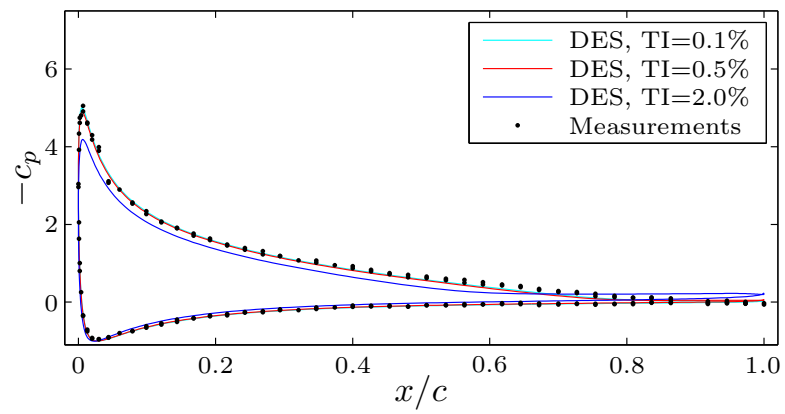

(a) $A O A=12^{\circ}$

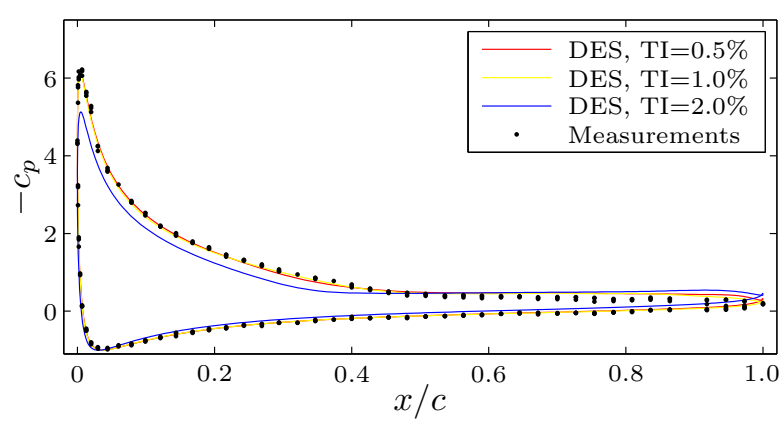

(c) $A O A=15^{\circ}$

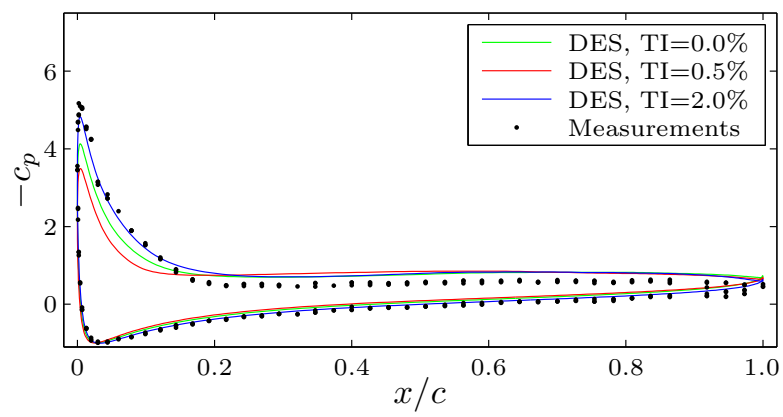

(e) $A O A=18^{\circ}$

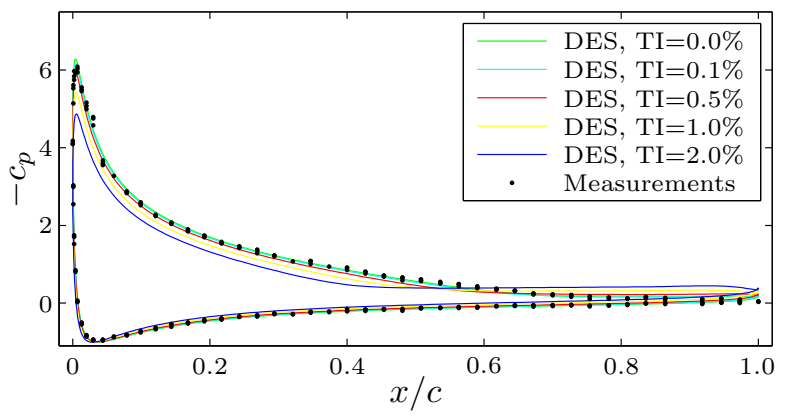

(b) $A O A=14^{\circ}$

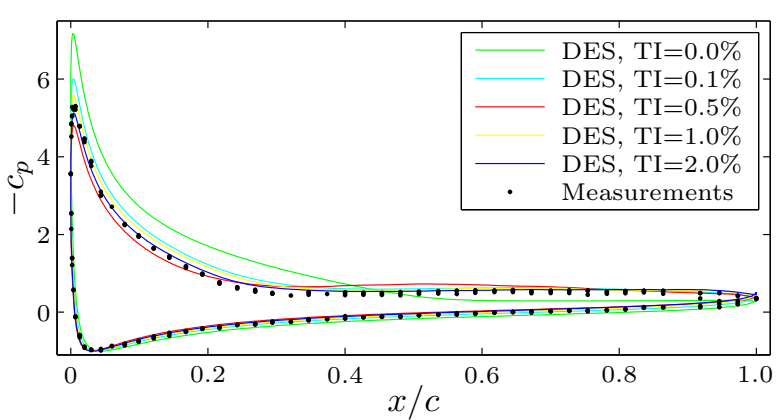

(d) $A O A=16^{\circ}$

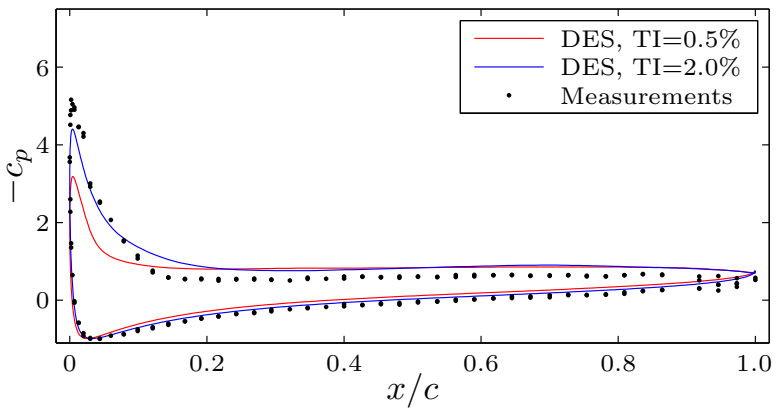

(f) $A O A=20^{\circ}$

Figure 9. Surface pressure coefficient.

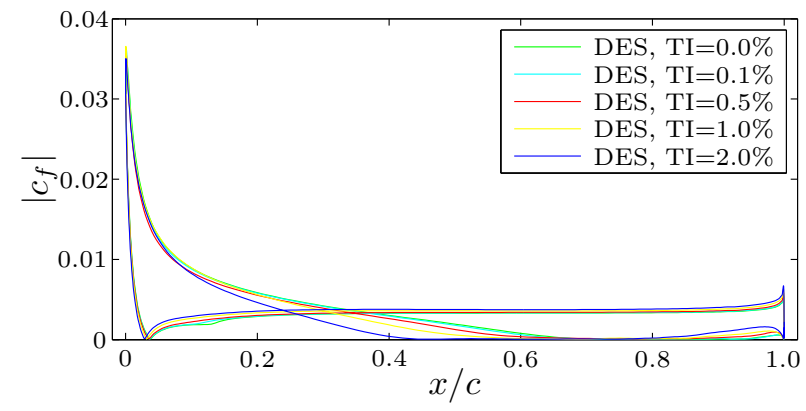

(a) $A O A=14^{\circ}$

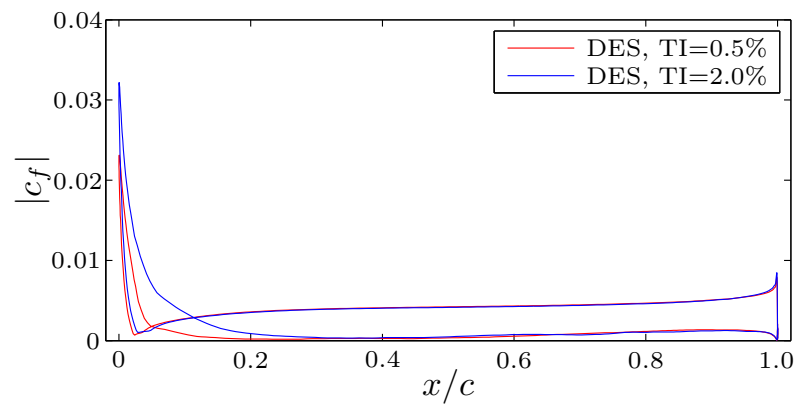

(b) $A O A=20^{\circ}$

Figure 10. Skin friction coefficient. 


\section{B. Flow Development and Time Averaging}

The required time for flow development and averaging is very different for the simulations. In general, the flow development time is largest for angles of attack close to stall and for low to moderate turbulence intensities.

In Figure 11 the lift coefficient is plotted versus nondimensional time for the inflow turbulence intensities $0.1 \%, 0.5 \%$ and $2.0 \%$. With the high TI the flow develops quickly and becomes a stationary stochastic process; after about 10 time units the flow is stable with constant mean lift. The simulation of the high TI flow is stopped after about 55 time units. The flow with $T I=0.5 \%$ develops slowly and the mean does not become stationary before 45 time units. Here a large separated region moves around on the airfoil, which causes the lift coefficient to vary slowly and with large amplitude. A similar separated region can be seen in the flow with $T I=0.1 \%$.

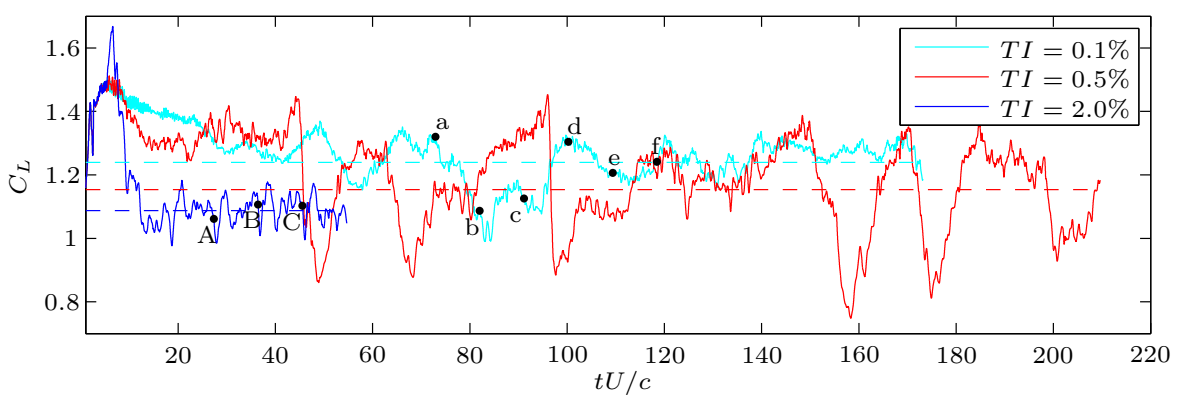

Figure 11. Time series of lift coefficient for $\mathrm{AOA}=16^{\circ}$. Dashed lines are averages after flow development phase. In Figure 12 the flow with $\mathbf{T I}=\mathbf{0 . 1} \%$ is visualized at the six instances marked by $\mathrm{a}-\mathrm{f}$ and in Figure 13 the flow with $2.0 \%$ is visualized at the instances $\mathrm{A}-\mathrm{C}$.

In Figure 11 six instances are marked by a-f, and at these instances the flow is visualized in Figure 12. Here the instantaneous isovorticity surfaces and surface limited streamlines show where the flow is attached and where it is separated. It is seen that the flow is very unsteady and the variation in the spanwise direction is large. The importance of modeling the full width of the wind tunnel is clearly illustrated.
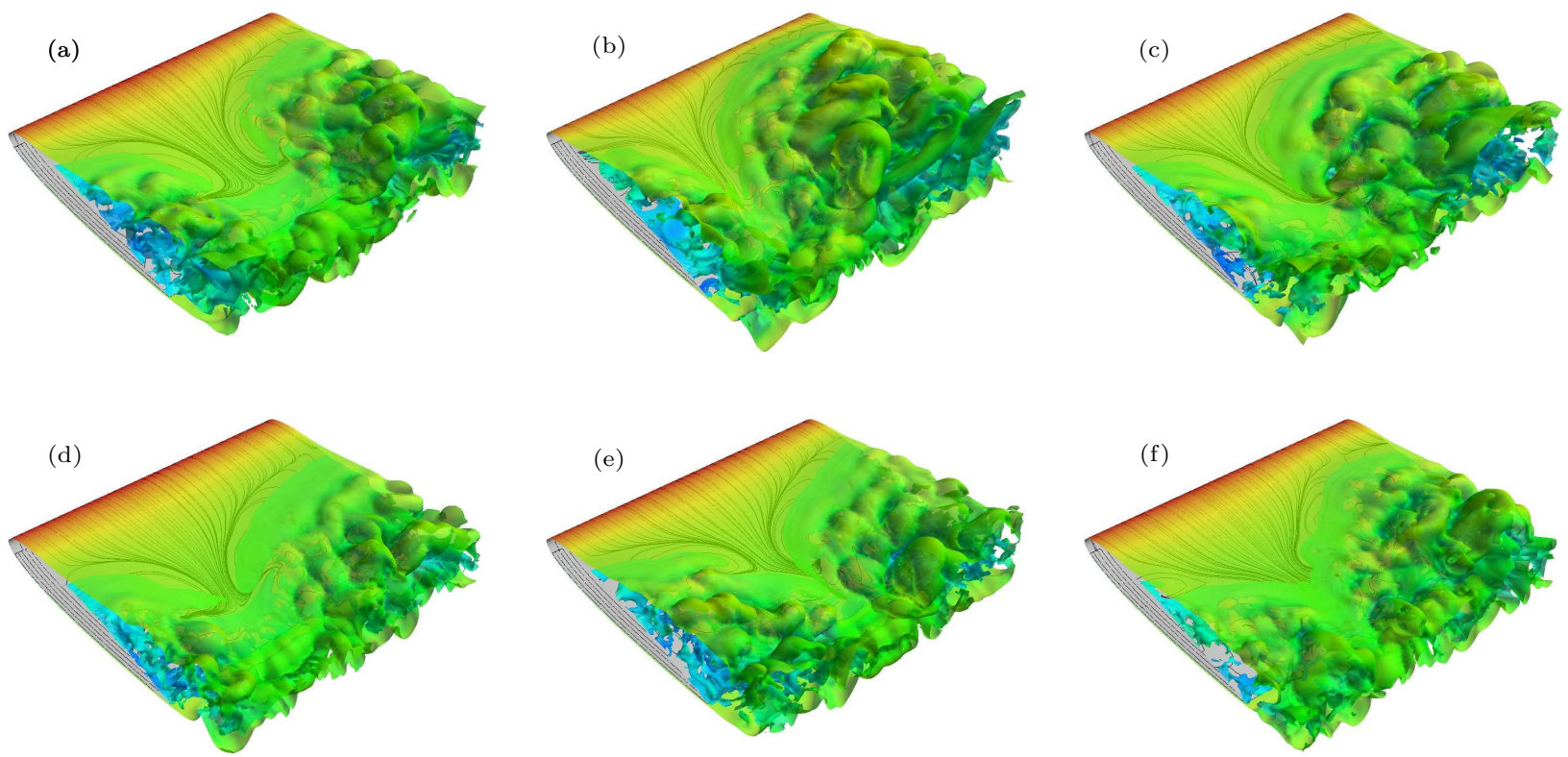

Figure 12. Instantaneous surface limited streamlines and isovorticity colored by streamwise velocity. The lift coefficient at the six instants (a)-(f) is marked on Figure 11. The AOA is $16^{\circ}$ and the TI is $0.1 \%$.

As expected it can be observed that the lift coefficient is high when the flow is attached over large parts of the airfoil. At instant (a) there is a large tongue of attached flow which gives a high lift. At (b) the 
separation has moved forward and closed most of tongue causing a large drop in the lift. In the remaining snapshots it can be seen how lift the fluctuates with the flow.

In Figure 13 the isovorticity and surface limited streamlines are shown at three instances for $2.0 \%$ TI. The time separation between the instances is the same as in Figure 12. It is seen that the separation point shows smaller variations in time and spanwise direction compared to the simulation with $0.1 \%$ TI. This is the cause of the low variation in lift coefficient.
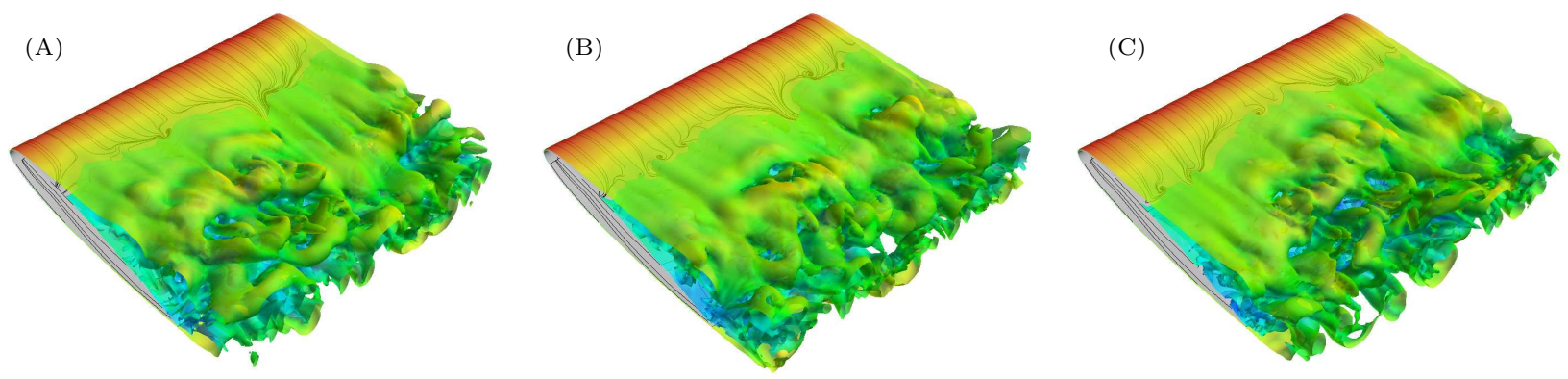

Figure 13. Instantaneous surface limited streamlines and isovorticity colored by streamwise velocity. The lift coefficient at the three instants $(\mathrm{A})-(\mathrm{C})$ is marked on Figure 11 . The AOA is $16^{\circ}$ and the TI is $2.0 \%$.

\section{Resolved Stresses and Eddy Viscosity}

The resolved turbulence imposed on the inflow boundary decays slightly as it is convected from the inflow boundary to the airfoil. The intensity of resolved turbulence is shown in Figure 14. It has been averaged in time and over the free cross section area of the tunnel (i.e. the cross section area excluding the airfoil).

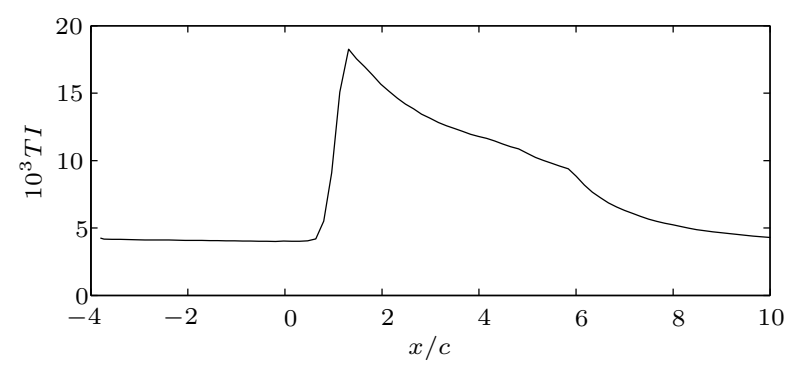

Figure 14. Average turbulence intensity for $\mathrm{AOA}=12^{\circ}$ and $\mathrm{TI}=0.5 \%$ on the inflow boundary. The leading edge of the airfoil is located at $x / c=0$ and the cells are stretched for $x / c \geq 6$.

It is observed that the resolved TI is slightly lower than what is imposed on the inflow boundary. That is, some resolved energy is lost in the very first part of the domain as was also shown in Figure 3 . Turbulence is generated in the separated region and in the wake of the airfoil. The intensity peaks around $c / 4$ downstream of the trailing edge. From there the turbulence decays exponentially, until the stretching of cells begins around $x / c=6$.

Figure 15 shows the average eddy viscosity close to the airfoil for the flow at $12^{\circ} \mathrm{AOA}$ and $0.5 \%$ turbulence intensity. It has been averaged over time and the spanwise direction. Far from the airfoil the eddy viscosity has a nearly constant value of 3.3 times the molecular viscosity. There is a region both along the pressure and suction side where the turbulent viscosity is increased. This is expected and appears because the production term is large here due to the large velocity gradient. In the blue zone reaching from the stagnation point along the suction side of the airfoil the eddy viscosity is lower, which is due to a limit ${ }^{12}$ on the production term in the $k$ equation. The production is limited in a zone upstream of the leading edge and the low viscosity is convected downstream. As expected, the eddy viscosity is high in the wake and in the separated region.

In Figure 16 the subgrid kinetic energy from the $k-\omega$ SST model is shown in the domain close to the airfoil. The five lines marked on the figure correspond to the ones in Figure 15 . Along line $1 k_{s g s}$ is low close to the wall. Here the flow is separated and in the separation bubble the velocity gradient is low and 


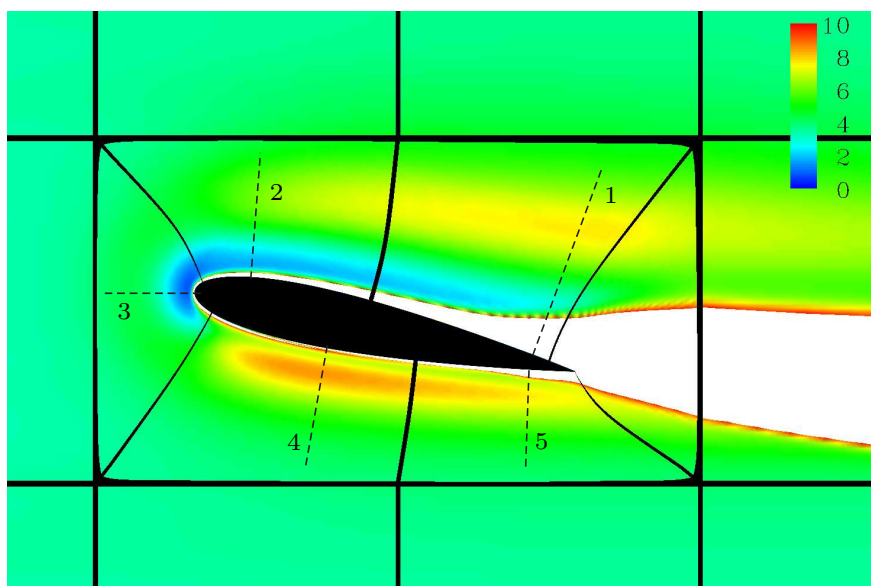

(a) $\nu_{t} / \nu$ in the domain. In the white area $\nu_{t}$ is higher than $10 \nu$. Solid black lines show the blocks of the grid. Dashed lines show the five positions used in the figure to the right. As can be seen from the figure to the right there is a white region close to the wall up to $n^{+} \approx 1000$ dependent on the position.

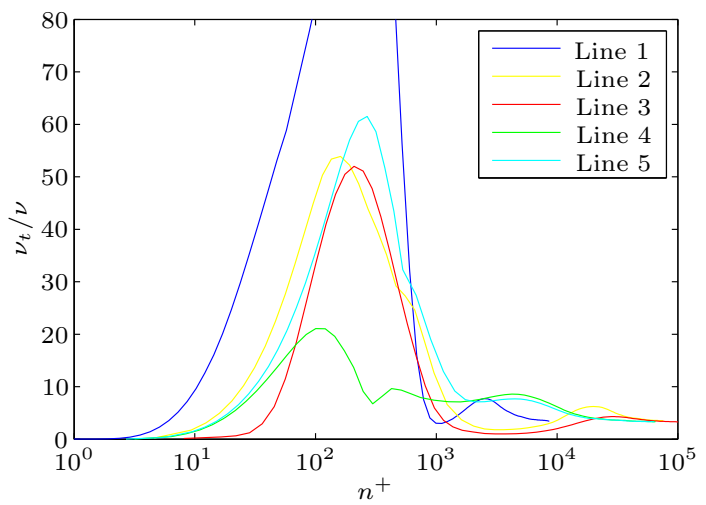

(b) $\nu_{t} / \nu$ along the five lines indicated to the left. Along line $1 \nu_{t} / \nu$ peaks at $n^{+} \approx 250$ with a value of 200 .

Figure 15. Averaged eddy viscosity $\nu_{\mathrm{t}}$ normalized by the molecular viscosity $\nu$. The AOA is $12^{\circ}$ and TI is $0.5 \%$.

consequently the production term in the $k$ equation is low. Close to the leading edge on the suction side (line 2) the velocity gradient is high and as expected the subgrid kinetic energy is high as well. At position 3 there is a local minimum at $n^{+} \approx 30$. This is caused by convection of low $k_{\text {sgs }}$ from the stagnation point. Far from the wall $k_{s g s} / U_{\infty}^{2}$ is approximately $10^{-6}$.

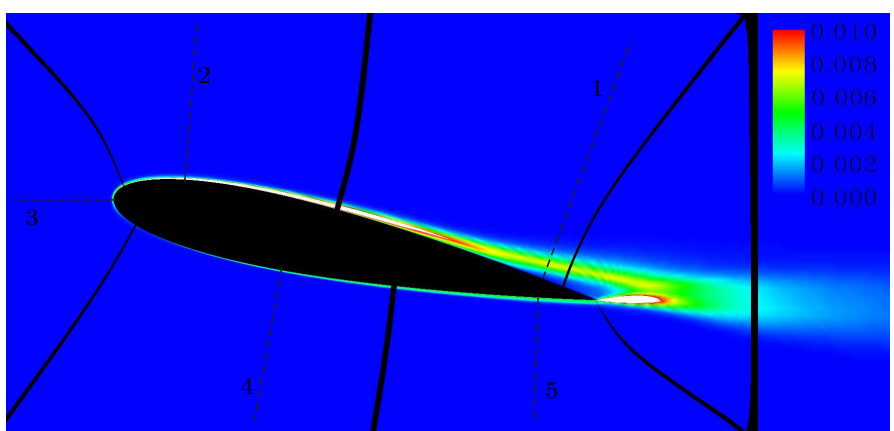

(a) $k_{s g s}$ in the domain close to the airfoil. In the white area $k_{s g s}$ is higher than $10^{-2} U_{\infty}^{2}$. Solid black lines show the blocks of the grid. Dashed lines show the five positions used in the figure to the right.

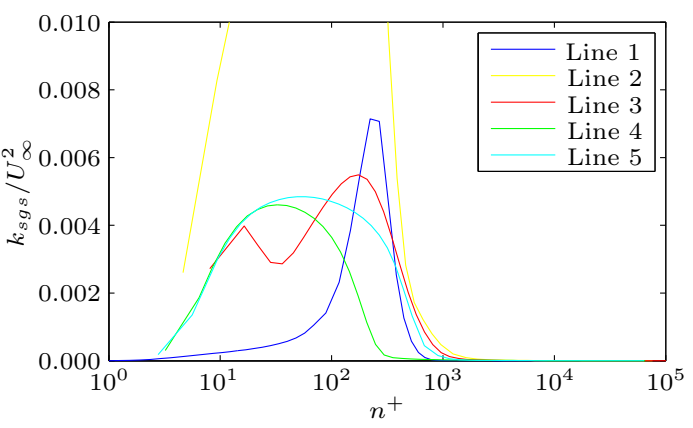

(b) $k_{\text {sgs }}$ along the five lines indicated to the left. Along line $2 k_{\text {sgs }}$ peaks at $n^{+} \approx 125$ with a value of $14 U_{\infty}^{2}$.

Figure 16. Averaged subgrid kinetic energy, $\mathrm{k}_{\mathrm{sgs}}$. The $\mathrm{AOA}$ is $12^{\circ}$ and $\mathrm{TI}$ is $0.5 \%$.

In Figure 17 the resolved kinetic energy is shown close to the airfoil. At line 1 the resolved kinetic energy is high, as the line intersects the separation bubble. Far from the airfoil the resolved kinetic energy has a constant value of $2.5 \times 10^{-4} U_{\infty}^{2}$ corresponding to a resolved turbulence intensity of $0.41 \%$.

By comparing Figures 16 and 17 it can be observed that the subgrid kinetic energy is high close to the wall and in the beginning of the wake. The resolved kinetic energy is high in the wake (peak value of $\left.2.2 \times 10^{-2} U_{\infty}^{2}\right)$ but also far from the airfoil, where the subgrid kinetic energy is low. 


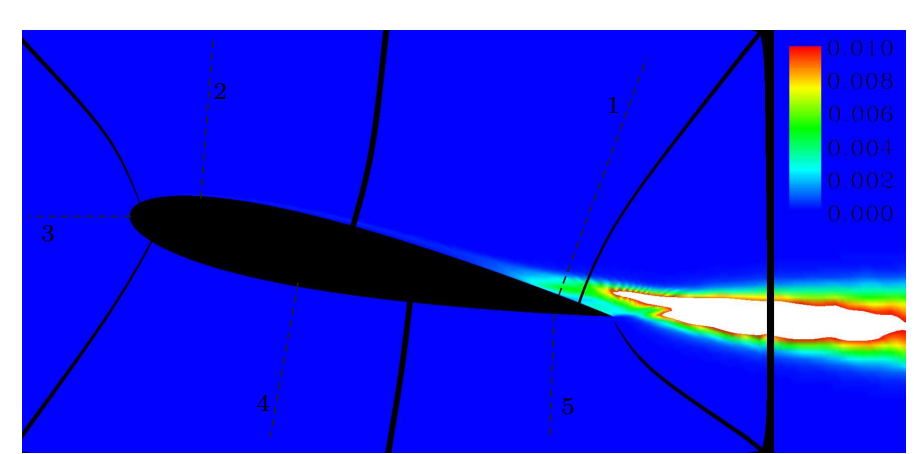

(a) $k_{\text {res }}$ in the domain close to the airfoil. In the white area $k_{r e s}$ is higher than $10^{-2} U_{\infty}^{2}$. Solid black lines show the blocks of the grid. Dashed lines show the five positions used in the figure to the right.

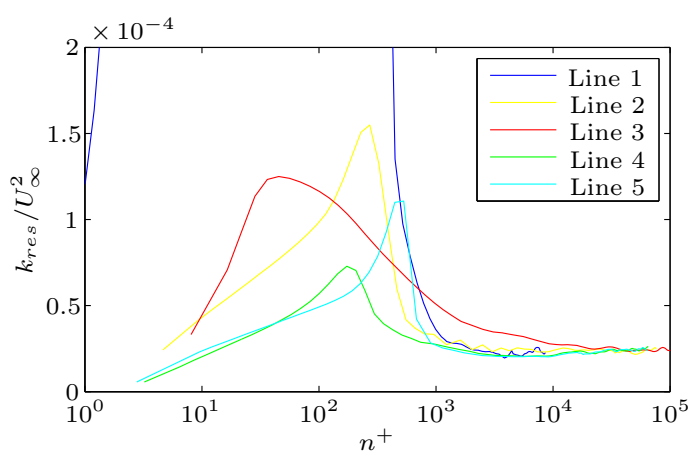

(b) $k_{\text {res }}$ along the five lines indicated to the left. Along line $1 k_{r e s}$ is high in the separation bubble. The maximum value is $5 \times 10^{-3} U_{\infty}^{2}$ at $n^{+} \approx 150$.

Figure 17. Averaged resolved kinetic energy, $k_{r e s}$. The AOA is $12^{\circ}$ and TI is $0.5 \%$.

\section{Conclusions}

A technique for generating synthetic turbulence is presented. The generated field must contain all three components of the velocities to minimize the need for a long precursor simulation. It is, however, suggested to run a shorter precursor simulation with the generated turbulence to allow the incorrect phase information to be corrected. Further, the subgrid parameters will then adjust to the velocity field and the computational setup. After the precursor simulation the turbulence can be imposed on the inflow boundary condition and will then decay according to empirical data.

From the study of the airfoil flow it is concluded that the flow is sensitive to the intensity of the resolved inflow turbulence. The comparison of simulations with and without inflow turbulence to experimental results shows that the simulations with inflow turbulence agree better with the experiment. Without turbulence the flow is predicted to stall at too high angle of attack.

The simulated and measured pressure distributions show good agreement for resolved turbulence intensities close to the intensities measured in the wind tunnel. The turbulence intensity that gives best agreement with measurements is not constant for all angles of attack. At high angles of attack the agreement is better for higher turbulence intensities.

The effect of the resolved turbulence on the separation point depends on the angle of attack. For angles of attack lower than stall the separation point moves upstream when the turbulence intensity is increased. For angles of attack higher than stall the effect is the opposite. At $16^{\circ}$ angle of attack the flow is very sensitive to the resolved turbulence. The lift coefficient is predicted to be approximately $30 \%$ lower for TI $=2.0 \%$ compared to a simulation without resolved inflow turbulence.

The simulations of this paper are all performed assuming fully turbulent conditions without a model for laminar/turbulent transition. For the NACA 0015 airfoil the influence from laminar/turbulent transition is low. For this reason, the agreement of simulations with measurements is good. For airfoils with higher sensitivity to transition the simulations without a transition model would produce results in poor agreement with measurements. The inclusion of the laminar/turbulent transition mechanism, especially in connection with by-pass transition, as for the present cases with high free stream turbulence, is not straightforward and will be the subject for further studies.

\section{Acknowledgements}

This work was funded by "The Danish Council for Strategic Research" under the project "Airfoils in Turbulent Inflow". The authors gratefully acknowledge the experimental data supplied by LM Glasfiber. The simulations have been carried out on Alfheim - A linux cluster located at DTU and financed by Danish Center for Scientific Computing (DCSC) Grant nr. HDW-0107-02 from January 2007. 


\section{References}

${ }^{1}$ Spalart, P. R., Jou, W.-H., Strelets, M., and Allmaras, S. R., "Comments on the Feasibility of LES for Wings, and on a Hybrid RANS/LES Approach," 1st AFOSR International Conference on DNS/LES, edited by C. Liu and Z. Liu, Greyden Press, Columbus OH, August 1997, pp. 137-147.

${ }^{2}$ Strelets, M., "Detached Eddy Simulation of Massively Separated Flows," 39th AIAA Aerospace Sciences Meeting and Exhibit, AIAA Paper 2001-0879, Reno, NV, January 8-11 2001.

${ }^{3}$ Shur, M., Spalart, P. R., Strelets, M., and Travin, A., "Detached-Eddy Simulation of an Airfoil at High Angle of Attack," Engineering Turbulence Modelling and Experiments, Vol. 4, 1999, pp. 669-678.

${ }^{4}$ Bertagnolio, F., Sørensen, N. N., and Johansen, J., "Profile Catalogue for Airfoil Sections Based on 3D Computations," Tech. Rep. Ris $\varnothing$-R-1581(EN), RIS $\varnothing$ National Laboratory, Roskilde, 2006.

${ }^{5}$ Johansen, J., Sørensen, N. N., Michelsen, J. A., and Shreck, S., "Detached Eddy Simulation of Flow Around the NREL Phase VI Blade," Wind Energy, Vol. 5, 2002, pp. 185-197.

${ }^{6}$ Fuglsang, P. and Bove, S., "Wind Tunnel Testing Of Airfoils Involves More Than Just Wall Corrections," Proceedings of European Wind Energy Conference and Exhibition 2008, Brussels, March 31-April 32008.

${ }^{7}$ Døssing, M., "High Frequency Microphone Measurements for Transition Detection on Airfoils," Tech. Rep. Risø-R1645(EN), RIS $\varnothing$ National Laboratory - DTU, Roskilde, 2008.

${ }^{8}$ Bove, S., "LSWT Report of Campaign NACA 0015_1," Tech. rep., LM Glasfiber, Lunderskov, 2008.

${ }^{9}$ Michelsen, J. A., "Basis3D - A Platform for Development of Multiblock PDE Solvers," Tech. Rep. AFM 92-05, Technical University of Denmark, Lyngby, 1992.

${ }^{10}$ Michelsen, J. A., "Block Structured Multigrid Solution of 2D and 3D Elliptic PDEs," Tech. Rep. AFM 94-06, Technical University of Denmark, Lyngby, 1994.

${ }^{11}$ Sørensen, N. N., General Purpose Flow Solver Applied to Flow over Hills, Ph.D. thesis, RIS $\varnothing$ National Laboratory, Roskilde, 1995, Ris $\varnothing-\mathrm{R}-827(\mathrm{EN})$.

${ }^{12}$ Menter, F. R., "Zonal Two Equation $k-\omega$ Turbulence Models for Aerodynamic Flows," 24th Fluid Dynamics Conference, AIAA paper no. 93-2906, Orlando, FL, July 6-9 1993.

${ }^{13}$ Spalart, P. R., Deck, S., Shur, M. L., Squires, K. D., Strelets, M. K., and Travin, A., "A New Version of DetachedEddy Simulation, Resistant to Ambiguous Grid Densities," Theoretical and Computational Fluid Dynamics, Vol. 20, 2006, pp. 181-195.

${ }^{14}$ Mann, J., "Wind Field Simulation," Probabilistic Engineering Mechanics, Vol. 13, No. 4, 1998, pp. 269-282.

${ }^{15}$ Shinozuka, M. and Jan, C. M., "Digital Simulation of Random Processes and its Application," Journal of Sound and Vibration, Vol. 25, No. 1, 1972, pp. 111-128.

${ }^{16}$ Bechmann, A., Large-Eddy Simulation of Atmospheric Flow over Complex Terrain, Ph.D. thesis, Ris $\emptyset$ National Laboratory, Roskilde, November 2006.

${ }^{17}$ Comte-Bellot, G. and Corrsin, S., "The Use of a Contraction to Improve the Isotropy of Grid-Generated Turbulence," Journal of Fluid Mechanics, Vol. 25, No. 4, 1966, pp. 657-682.

${ }^{18}$ Sørensen, N. N., "CFD modeling of laminar-turbulent transition for airfoils and rotors," 42nd AIAA aerospace sciences meeting and exhibit. 26th AIAA applied aerodynamics conference, AIAA Paper 2008-7323, Honolulu, HI, August 18-21 2008. 